\title{
Fluorescence Spectroscopy Study on the Interaction between Evodiamine and Bovine Serum Albumin
}

\author{
Mingxiong Tan, ${ }^{1,2}$ Weijiang Liang, ${ }^{1}$ Xujian Luo, ${ }^{1}$ and Yunqiong Gu ${ }^{1}$ \\ ${ }^{1}$ School of Chemistry and Material, Yulin Normal University, Yulin 537000, China \\ ${ }^{2}$ The Key Laboratory for the Chemistry and Molecular Engineering of Medicinal Resources, \\ School of Chemistry \& Chemical Engineering, Guangxi Normal University, Guilin 541004, China \\ Correspondence should be addressed to Mingxiong Tan; tanmx00@163.com
}

Received 14 June 2012; Accepted 3 December 2012

Academic Editor: Tomokazu Yoshimura

Copyright (C) 2013 Mingxiong Tan et al. This is an open access article distributed under the Creative Commons Attribution License, which permits unrestricted use, distribution, and reproduction in any medium, provided the original work is properly cited.

\begin{abstract}
The interaction of evodiamine (Evo) with bovine serum albumins (BSAs) at different two temperatures (298 and $310 \mathrm{~K})$ was investigated by means of fluorescence spectroscopy. The experimental results showed that Evo binds with BSA via a static quenching procedure with association constants $K$ of $1.61 \times 10^{6} \mathrm{~L} / \mathrm{mol}$ at $298 \mathrm{~K}$ and $6.78 \times 10^{5} \mathrm{~L} / \mathrm{mol}$ at $310 \mathrm{~K}$. The number of bound Evo molecules per protein is 1.31 at $298 \mathrm{~K}$ and 1.33 at $310 \mathrm{~K}$. The results suggested that Evo reacts with BSA chiefly through hydrophobic and electrostatic interactions, and it does not alter the $\alpha$-helical nature of BAS.
\end{abstract}

\section{Introduction}

Evodiamine (Figure 1), a quinolone alkaloid, is the major component isolated from the fruit of Evodia rutaecarpa, which is a traditional medicinal plant distributed in East Asia, especially in China, and has been used for a long time as a traditional Chinese medicine for the treatment of gastrointestinal disorders, headache, and postpartum hemorrhage. In pharmacology studies, it has been reported that evodiamine (Evo) was found to have antinociceptive, antiinflammatory, antiobesity, vasodilatory, thermoregulatory, analgesic, cardiotonic, uterotonic, and antitumor activities [1-3]. Studies concerning screening of alkaloids have shown that evodiamine exhibits the strongest cytotoxicity activity against human colon and hepatoblastoma cell lines and inhibitory activity on human colon carcinoma cell. Further studies demonstrate that evodiamine has antitumor potential by inhibiting proliferation, inducing apoptosis and reducing invasion and metastasis of a wide variety of tumor cells, including breast cancer cells, prostate cancer cells, leukemic T-lymphocyte cells, melanoma cells, cervical cancer cells, colon cancer cells, and lung cancer cells. More importantly, evodiamine not only sensitizes chemoresistant breast cancer cells to adriamycin, but also shows little toxicity against normal human peripheral blood cells [4].
Serum albumin (SA) is a multiple function protein and acts as the transporter and disposition of many endogenous and exogenous ligands, including fatty acids, amino acids, metals ions, and numerous pharmaceuticals by means of hydrogen bonding, hydrophobic, electrostatic, and metal interactions [4-7]. The interaction intensity between antitumor drugs and SA may effect on their bioavailability and toxicity [8-10]. In this regard, bovine serum albumin (BSA) has been studied extensively, partly because of its structural homology with human serum albumin (HSA). BSA is composed of three linearly arranged, structurally homologous subdomains. It has two tryptophan residues that possess intrinsic domains, and each domain in turn is the product of two fluorescence: $\operatorname{Trp} 134$, which is located on the surface of subdomain IB, and Trp212, locating within the hydrophobic binding pocket of subdomain IIA. The binding sites of BSA for endogenous and exogenous ligands may be in these domains.

Fluorescence quenching is considered as a method for measuring binding affinities. Fluorescence quenching is the decrease of the quantum yield of fluorescence from a fluorophore induced by a variety of molecular interactions with quencher molecule $[11,12]$. It is, therefore, of interest to use quenching of the intrinsic tryptophan fluorescence of BSA 


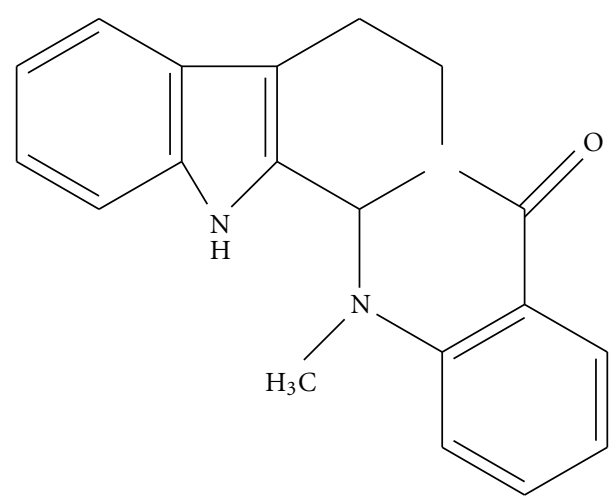

Figure 1: The structure of Evodiamine.

(Trp-212 and Trp-134) as a tool to study the interaction of BSA and evodiamine (Evo) $[13,14]$.

\section{Results and Discussion}

2.1. Fluorescence Quenching. BSA has two tryptophan residues that possess intrinsic fluorescence: Trp-134 in the first domain and Trp-212 in the second domain. Tryptophan emission dominates BSA fluorescence spectra in the UV region. $[15,16]$. When other molecules interact with $\mathrm{BSA}$, tryptophan fluorescence may change depending on the impact of such interaction on the protein conformation. The fluorescence intensity of BSA-Evo system was measured with a $\mathrm{pH}$ of 7.34 and two different temperatures of 298 and $310 \mathrm{~K}$. The effects of Evo on the fluorescence of BSA at temperatures of $298 \mathrm{~K}$ and $310 \mathrm{~K}$ are shown in Figure 2. The intensity of the characteristic broad emission band at $377 \mathrm{~nm}$ decreases markedly with the increasing concentration of Evo, indicating that an interaction between Evo and BSA has occurred, and the variation in intensity may result from the changed protein conformation or direct quenching effect by Evo [17, 18]. However, the maximum emission wavelength of BSA barely changed during the interaction. As a result, we predict that polyamine binds mainly with the two fluorophores Trp-212 buried inside and Trp-134 located on the surface of BSA, indicating that Trp-212 located within a hydrophobic binding pocket of the protein is not exposed to any change in polarity [19]. Similarly, Trp 134 located in this subdomain region would probably place it in the hydrophobic packing interaction between helices and close to the "distal" opening of the IB site. This suggests that the evodiamine is binding in the "proximal" IB side, and the same binding mode of large heterocyclic molecules similar to camptothecin with HSA is described in the published literature [20].

Synchronous fluorescence spectra show Trp residues of BSA only at the wavelength interval $\Delta \lambda$ of $60 \mathrm{~nm}$ and Tyr residues of BSA only at $\Delta \lambda$ of $15 \mathrm{~nm}$. Synchronous fluorescence spectra of BSA-Evo are shown in Figure 3. It is apparent that the intensity of Trp or Tyr residues decreases in the presence of Evo. However, the emission peak position of Trp or Tyr residues does not show significant shift, indicating that the polarity around Trp or Tyr residues is unchangeable [21].
So, Evo does not obviously affect the $\alpha$-helical conformation of BSA.

2.2. Binding Parameters. Florescence quenching data was analyzed to obtain various binding parameters for the interaction of Evo and BSA. The procedure of the fluorescence quenching was first assumed to be a dynamic quenching process.

The dynamic quenching constant $K_{\mathrm{sv}}$ and the apparent bimolecular quenching rate constant $K_{q}$ were calculated with the following Stern-Volmer equation [22]:

$$
\frac{F_{0}}{F}=1+K_{q} \tau_{0}[Q]=1+K_{\mathrm{sv}}[Q]
$$

where $F_{0}$ and $F$ are the relative fluorescence intensities in the absence and presence of quencher, $[Q]$ is the concentration of quencher, $K_{\mathrm{sv}}$ is the Stern-Volmer dynamic quenching constant, $k_{q}$ is the bimolecular quenching rate constant, and $\tau_{0}$ is the average bimolecular lifetime in the absence of quencher evaluated at about $5 \mathrm{~ns}$. The plot of $F_{0} / F$ versus [Q] gives a straight line, and $K_{\mathrm{sv}}$ is thus obtained from the slope. Plots of $F_{0} / F$ versus [Q] are shown in Figure 4, and the calculated $K_{\mathrm{sv}}$ and $k_{q}$ are listed in Table 1 . The values of $k_{q}$ obtained are $4.86 \times 10^{12} \mathrm{~L} \mathrm{~mol}^{-1} \mathrm{~s}^{-1}$ at $298 \mathrm{~K}$ and $2.41 \times 10^{12} \mathrm{~L} \mathrm{~mol}^{-1} \mathrm{~s}^{-1}$ at $310 \mathrm{~K}$, which are much larger than the maximum scattering collisional quenching constant of various quenchers of $2.0 \times 10^{10} \mathrm{~L} \mathrm{~mol}^{-1} \mathrm{~s}^{-1}$, indicating that the probable quenching mechanism of the intrinsic fluorescence of BSA was not initiated by a dynamic process but a static quenching procedure [23].

The apparent association constant $K$ and the number of binding site $n$ were calculated using [22]

$$
\log \left[\frac{\left(F_{0}-F\right)}{F}\right]=\log K+n \log [Q],
$$

where $K$ and $n$ are the apparent association constant and the number of binding sites. Plots of $\log \left[\left(F_{0}-F\right) / F\right]$ versus $\log$ [Q] are shown in Figure 5. $K$ and $n$ were thus obtained from the intercept on the $y$-axis and the slope, respectively. The calculated $K$ and $n$ for Evo at two temperatures are listed in Table 2 . The medium association constants of $K\left(1.61 \times 10^{6}\right.$ 


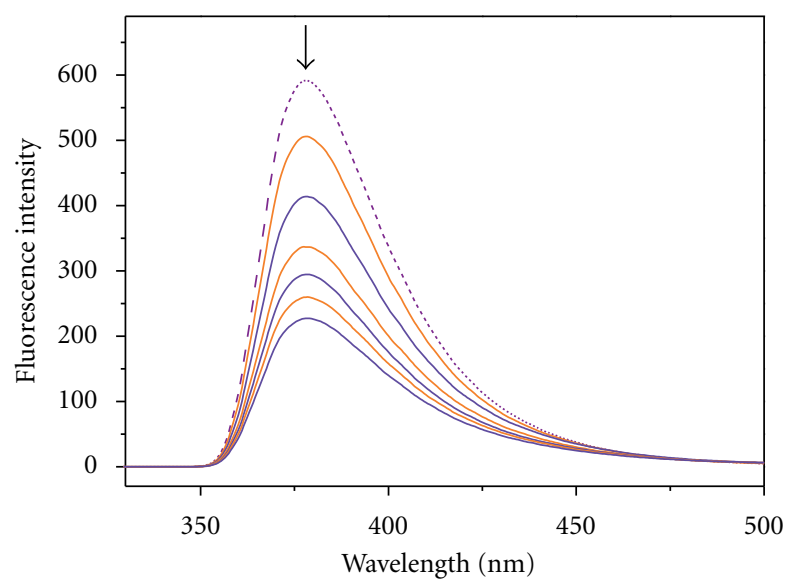

(a)

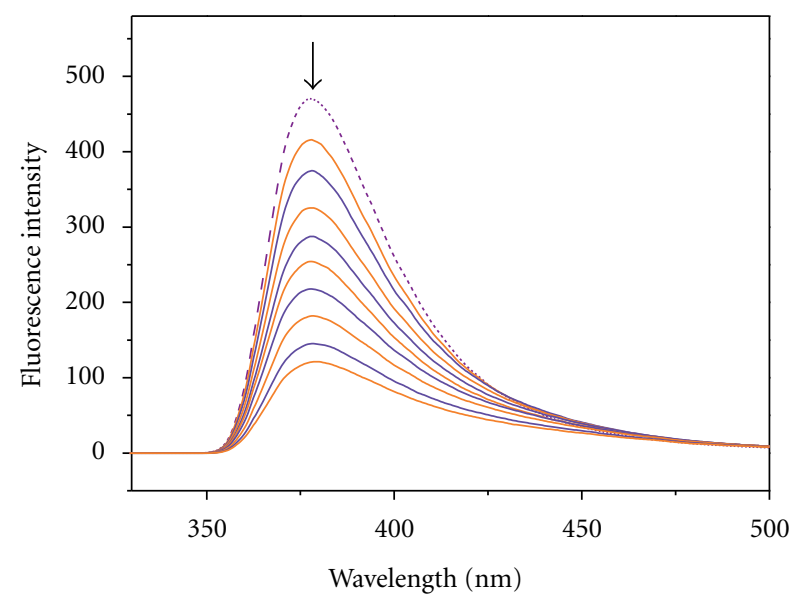

(b)

Figure 2: Emission spectra of BSA with various amounts of Evo at $298 \mathrm{~K}$ (a) and $310 \mathrm{~K}$ (b), $C_{\mathrm{BSA}}(\mu \mathrm{M}): 10, C_{\mathrm{Evo}}(\mu \mathrm{M}): 4,8,12,16,20,24,28$, 32 , and 36.

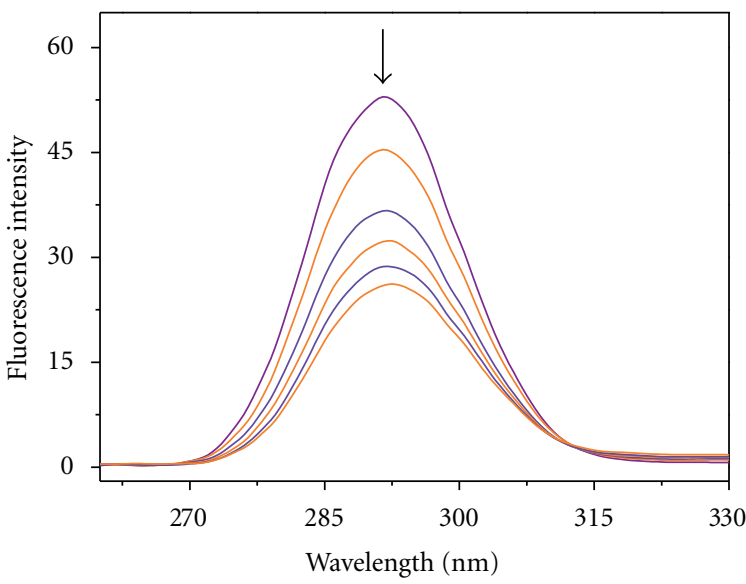

(a)

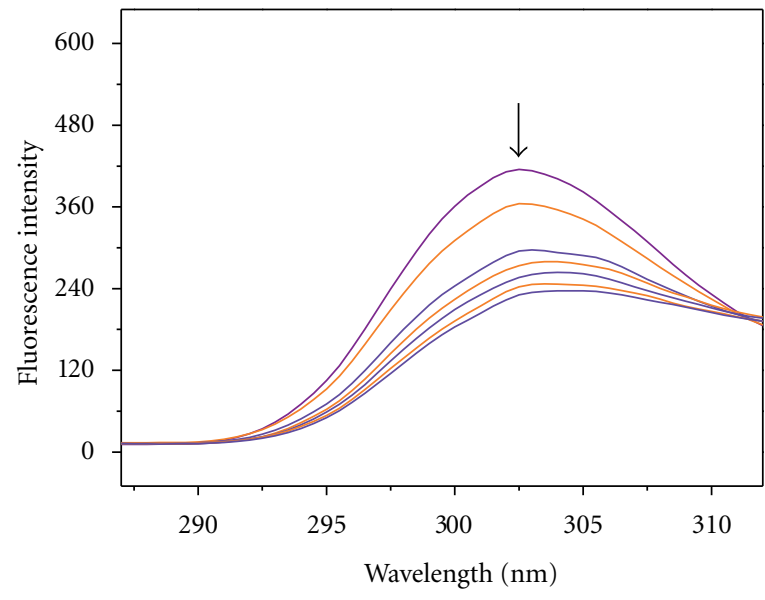

(b)

FIGURE 3: Synchronous fluorescence spectra of BSA with various amounts of Evo, (a) $\Delta \lambda=15 \mathrm{~nm}$, (b) $\Delta \lambda=60 \mathrm{~nm}$. $C_{\mathrm{BSA}}(\mu \mathrm{M}): 10$ and $C_{\text {Evo }}$ $(\mu \mathrm{M}): 4,8,12,16,20,24$, and $28 . T=298 \mathrm{~K}$.

at $298 \mathrm{~K}$ and $6.78 \times 10^{5}$ at $310 \mathrm{~K}$ ) suggest that the affinity of Evo for BSA is just at a moderate level compared with the reported binding constants of $10^{4}-10^{8}$, in which Sandip et al. have reported that serum albumin has a limited number of binding sites for endogenous and exogenous ligands that are typically bound reversibly [24]. For example, baicalein which is bound to BSA in the presence of $\mathrm{Fe}^{3+}$ has a lower affinity $\left(4.85 \times 10^{4} \mathrm{~L} / \mathrm{mol}\right)$ [15], while epicatechin gallate binds to BSA in a higher binding constant of $6.6 \times 10^{7} \mathrm{~L} / \mathrm{mol}$ [25]. The values of $n$ are 1.31 at $298 \mathrm{~K}$ and 1.33 at $310 \mathrm{~K}$, suggesting that 1-2 Evo molecules bound with BSA per protein. It is indicated that this number $(n)$ of binding site does not depend on the association constant $(K)$.

2.3. Fluorescence Resonance Energy Transfer. The rate of energy transfer depends on the extent of the overlapping of BSA, the donor emission spectrum with the acceptor absorption spectrum, the relative orientation of the donor
TABLE 1: Stern-Volmer quenching constants for BSA interaction with Evo, $R$ is the correlation coefficient.

\begin{tabular}{ccccc}
\hline & $T(\mathrm{~K})$ & $\begin{array}{c}K_{\mathrm{sv}} \\
\left(\times 10^{4} \mathrm{~L} / \mathrm{mol}\right)\end{array}$ & $\begin{array}{c}K_{q} \\
\left(\times 10^{12} \mathrm{~L} \mathrm{~mol}^{-1} \mathrm{~s}^{-1}\right)\end{array}$ & $R$ \\
\hline \multirow{2}{*}{ Evo-BSA } & 298 & 4.96 & 4.86 & 0.9874 \\
& 310 & 2.41 & 2.41 & 0.9806 \\
\hline
\end{tabular}

TABLE 2: Binding parameters for BSA interaction with Evo, $R$ is the correlation coefficient.

\begin{tabular}{lcccc}
\hline & $T(\mathrm{~K})$ & $K(\mathrm{~L} / \mathrm{mol})$ & $n$ & $R$ \\
\hline \multirow{2}{*}{ Evo-BSA } & 298 & $1.61 \times 10^{6}$ & 1.31 & 0.9948 \\
& 310 & $6.78 \times 10^{5}$ & 1.33 & 0.9938 \\
\hline
\end{tabular}

and acceptor transition dipoles, and the distance between these molecules [26]. 


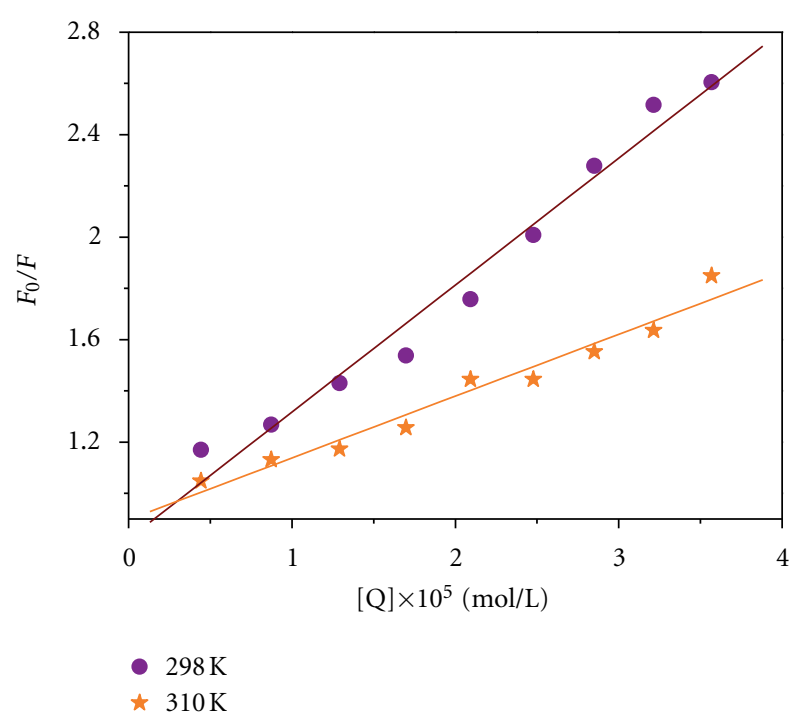

FIGURE 4: Sterne-Volmer plot of the fluorescence quenching of BSA with various amounts of Evo at $298 \mathrm{~K}(\bullet)$ and $310 \mathrm{~K}(\star)$.

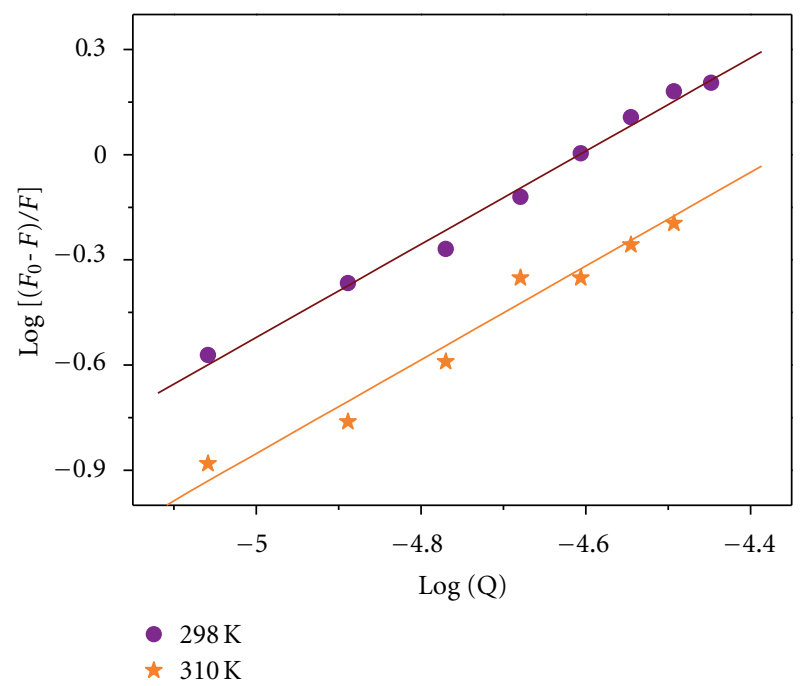

FIGURE 5: Logarithmic plot of the fluorescence quenching of BSA with various amounts of Evo at $298 \mathrm{~K}(\bullet)$ and $310 \mathrm{~K}(\star)$.

The energy transfer efficiency $(E)$ can be used to evaluate the distance $(r)$ between the ligands (acceptor) and BSA (donor) in protein by Förster's theory of dipole-dipole energy transfer as follows:

$$
E=1-\frac{F}{F_{0}}=\frac{R_{0}^{6}}{\left(R_{0}^{6}+r^{6}\right)},
$$

where $F$ and $F_{0}$ are the fluorescence intensity of BSA in the presence and absence of the acceptor, $r$ is the distance between the acceptor and donor, $R_{0}$ is the critical distance for $50 \%$ energy transfer which can be calculated using the following:

$$
R_{0}^{6}=8.8 \times 10^{-25} K^{2} N^{-4} \Phi J,
$$

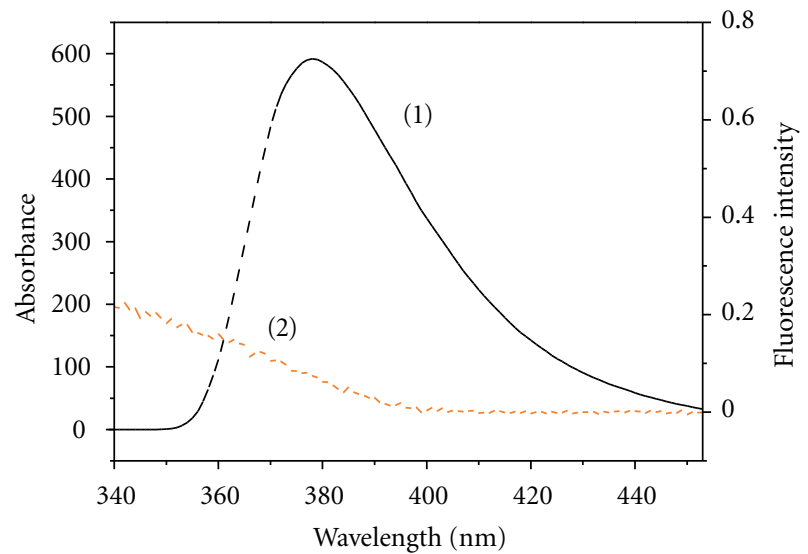

FIGURE 6: Overlap between the fluorescence emission spectrum of BSA (1) and UV absorption spectrum of Evo (2), $C_{\mathrm{BSA}}=C_{\mathrm{Evo}}=$ $10 \mu \mathrm{M}, T=298 \mathrm{~K}$.

TABLE 3: The calculated values of $J, r, R_{0}$, and $E$ of BSA with Evo, $R$ is the correlation coefficient.

\begin{tabular}{ccccc}
\hline & $J\left(\mathrm{~cm}^{3} \mathrm{~L} \mathrm{~mol}^{-1}\right)$ & $E(\%)$ & $R_{0}(\mathrm{~nm})$ & $r(\mathrm{~nm})$ \\
\hline Evo-BSA & $8.53 \times 10^{-14}$ & 0.41 & 1.67 & 1.57 \\
\hline
\end{tabular}

where the spatial orientation factor of the dipole $K^{2}=$ $2 / 3$, the refractive index of medium $N=1.36$, and the fluorescence quantum yield of donor $\varphi=0.15[27,28]$. $J$ can be evaluated by the overlap of the UV absorption spectra of acceptor with the fluorescence emission spectra of donor. $J$ is given by the following equation:

$$
J=\frac{\sum F(\lambda) \varepsilon(\lambda) \lambda^{4} \Delta \lambda}{\sum F(\lambda) \Delta \lambda} .
$$

The overlap between the BSA emission spectra with the absorption spectra of Evo is shown in Figure 6, and the calculated values of $J, r, R_{0}$, and $E$ are listed in Table 3. It is found that the $r$ value is $1.57 \mathrm{~nm}$ for Evo, which is less than the average distance of $2-8 \mathrm{~nm}$ between a donor and acceptor, indicating that the energy transfer occurred between BSA and Evo with great possibility $[8,29]$.

2.4. Thermodynamic Parameters. The thermodynamic parameters at different temperatures were analyzed to characterize the acting forces dominating the interaction. The enthalpy $(\Delta H)$, free energy $(\Delta G)$, and entropy $(\Delta S)$ changes are calculated according to the following Van't Hoff equation:

$$
\begin{gathered}
\ln \frac{K_{2}}{K_{1}}=\Delta H \frac{\left(1 / T_{1}-1 / T_{2}\right)}{R}, \\
\Delta G=\Delta H-T \Delta S, \\
\Delta G=-R T \ln K,
\end{gathered}
$$

where $K$ is the association constant at temperature $T$ and $R$ is the gas constant. The results are summarized in Table 4 . Four 
TABLE 4: The temperature-dependent thermodynamic parameters for the Evo-BSA system.

\begin{tabular}{ccccc}
\hline & $T(\mathrm{~K})$ & $\Delta G(\mathrm{~kJ} / \mathrm{mol})$ & $\Delta H(\mathrm{~kJ} / \mathrm{mol})$ & $\Delta S\left(\mathrm{~J} \cdot \mathrm{mol}^{-1} \mathrm{~K}^{-1}\right)$ \\
\hline \multirow{2}{*}{ Evo-BSA } & 298 & -2.32 & 60.13 & 209.59 \\
& 310 & -2.42 & 60.13 & 201.78 \\
\hline
\end{tabular}

types of acting forces including hydrogen bond, van der Waals force, electrostatic force, and hydrophobic interaction force may be involved in the interaction between a small molecule and a protein [30]. The positive value of $\Delta H$ and $\Delta S$ for Evo suggests that hydrophobic and electrostatic interactions may be involved in the association and contribute to these changes [31]. The negative value of $\Delta G$ indicates that the interaction of Evo with BSA is a spontaneous process.

\section{Conclusions}

In the paper, the fluorescence quenching mechanism and binding mode of evodiamine with bovine serum albumins were investigated. The experimental results showed that Evo binds with BSA via a static quenching procedure with association constants $K$ of $1.61 \times 10^{6} \mathrm{~L} / \mathrm{mol}$ at $298 \mathrm{~K}$ and $6.78 \times$ $10^{5} \mathrm{~L} / \mathrm{mol}$ at $310 \mathrm{~K}$. The number of bound Evo molecules per protein was 1.31 at $298 \mathrm{~K}$ and 1.33 at $310 \mathrm{~K}$. The results suggest that the Evo seems to react with BSA chiefly through hydrophobic and electrostatic interactions, and it does not alter the $\alpha$-helical nature of BAS. The distance $(r)$ between the ligands (acceptor) and BSA (donor) in protein is $1.57 \mathrm{~nm}$ for Evo, which is less than the average distance of $2-8 \mathrm{~nm}$ between a donor and acceptor, indicating that the energy transfer occurred between BSA and Evo with great possibility. The positive value of $\Delta H$ and $\Delta S$ for Evo suggests that hydrophobic and electrostatic interactions may be involved in the association and contribute to these changes. The negative value of $\Delta G$ indicates that the interaction of Evo with BSA is a spontaneous process.

\section{Experimental}

4.1. Materials. Evodiamine (Evo) was isolated from the unripe fruit of Evodia rutaecarpa (Juss.) Benth according to the methods of the literature [31]. The purity of Evo is $98 \%$ (HPLC). Bovine serum albumin (essentially fatty acid free) was purchased from Sino-American Biotech. Co. Ltd, Beijing. Tris-HCl-NaCl buffer solution ( $5 \mathrm{mM}$ Tris, $50 \mathrm{mM} \mathrm{NaCl}, \mathrm{pH}$ was digital adjusted to 7.34 by titration with hydrochloric acid with Sartorius professional meter, Tris = tri(hydroxymethyl) amino methane) was prepared using double-distilled water. Stock solution of BSA was prepared by dissolving it in Tris$\mathrm{HCl}$ buffer solution. Other chemicals were all of analytical grade, and doubly distilled water was used throughout the experiment.
4.2. Measurements. UV-Vis absorption spectra were performed on a Varian Cary100 UV-Visible spectrophotometer. Fluorescence measurements were performed on a FluoroMax-4 spectrophotometer.

4.2.1. UV-Vis Absorption Spectra. UV-Vis absorption spectra were performed on a Varian Cary100 UV-Visible spectrophotometer from $190 \mathrm{~nm}$ to $500 \mathrm{~nm}$.

4.2.2. Fluorescence Emission Titration. Fluorescence emission spectra were then measured at $298 \mathrm{~K}$ or $310 \mathrm{~K}$ with a $\mathrm{pH}$ of 7.34 and were recorded in a wavelength range of 290-500 nm. Samples were excited at $280 \mathrm{~nm}$. Fluorescence emission spectra of compounds were performed by using a fixed concentration of BSA $(10 \mu \mathrm{M})$ and increasing the concentration of Evo $(4,8,12,16,20,24,28,32$, and $36 \mu \mathrm{M})$.

4.2.3. The Synchronous Fluorescence Spectra. Synchronous fluorescence spectra were obtained by simultaneously scanning the excitation and emission monochromators. The wavelength interval $(\Delta \lambda)$ at 15 and $60 \mathrm{~nm}$ was recorded over a wavelength range of $240-360 \mathrm{~nm}$ by using a fixed concentration of BSA $(10 \mu \mathrm{M})$ and increasing the concentration of Evo $(4,8,12,16,20,24$, and $28 \mu \mathrm{M})$ at $298 \mathrm{~K}$.

\section{Acknowledgments}

The authors are grateful for the financial support from the National Natural Science Foundation of China (no. 21261025, 81060360), the Natural Science Foundation of Guangxi Province (no. 013064), the Key Laboratory for the Chemistry and Molecular Engineering of Medicinal Resources (Guangxi Normal University), and the Ministry of Education of China (no. CMEMR2011-09), as well as the talent training scheme of Guangxi Institutions of Higher Learning (2011-13-2).

\section{References}

[1] M. Ogasawara, T. Matsubara, and H. Suzuki, "Inhibitory effects of evodiamine on in vitro invasion and experimental lung metastasis of murine colon cancer cells," Biological and Pharmaceutical Bulletin, vol. 24, no. 8, pp. 917-920, 2001.

[2] J. Yamahara, T. Yamada, T. Kitani, Y. Naitoh, and H. Fujimura, "Antianoxic action of evodiamine, an alkaloid in Evodia rutaecarpa fruit," Journal of Ethnopharmacology, vol. 27, no. 1-2, pp. 185-192, 1989.

[3] W. F. Chiou, C. J. Chou, A. Y. C. Shum, and C. F. Chen, "The vasorelaxant effect of evodiamine in rat isolated mesenteric arteries: mode of action," European Journal of Pharmacology, vol. 215, no. 2-3, pp. 277-283, 1992.

[4] J. Jiang and C. Hu, "Evodiamine: a novel anti-cancer alkaloid from evodia rutaecarpa," Molecules, vol. 14, no. 5, pp. 1852-1859, 2009.

[5] T. Peters, All about Albumin: Biochemistry, Genetics and Medical Application, Academic Press, New York, NY, USA, 1996.

[6] D. C. Carter and X. M. He, "Structure of human serum albumin,” Science, vol. 249, no. 4966, pp. 302-303, 1990. 
[7] G. Weber, "Enumeration of components in complex systems by fluorescence spectrophotometry," Nature, vol. 190, no. 4770, pp. 27-29, 1961.

[8] L. Trynda-Lemiesz and M. Łuczkowski, "Human serum albumin: spectroscopic studies of the paclitaxel binding and proximity relationships with cisplatin and adriamycin," Journal of Inorganic Biochemistry, vol. 98, no. 11, pp. 1851-1856, 2004.

[9] L. L. Yan, X. Y. Wang, Y. Q. Wang, Y. M. Zhang, and Z. J. Guo, "Cytotoxic palladium(II) complexes of 8-aminoquinoline derivatives and the interaction with human serum albumin," Journal of Inorganic Biochemistry, vol. 106, no. 1, pp. 46-51, 2012.

[10] P. B. Kandagal, S. Ashoka, J. Seetharamappa, S. M. T. Shaikh, Y. Jadegoud, and O. B. Ijare, "Study of the interaction of an anticancer drug with human and bovine serum albumin: spectroscopic approach," Journal of Pharmaceutical and Biomedical Analysis, vol. 41, no. 2, pp. 393-399, 2006.

[11] P. Bourassa, C. D. Kanakis, P. Tarantilis, M. G. Pollissiou, and H. A. Tajmir-Riahi, "Resveratrol, genistein, and curcumin bind bovine serum albumin," Journal of Physical Chemistry B, vol. 114, no. 9, pp. 3348-3354, 2010.

[12] N. Tayeh, T. Rungassamy, and J. R. Albani, "Fluorescence spectral resolution of tryptophan residues in bovine and human serum albumins," Journal of Pharmaceutical and Biomedical Analysis, vol. 50, no. 2, pp. 107-116, 2009.

[13] V. Anbazhagan and R. Renganathan, "Study on the binding of 2,3-diazabicyclo[2.2.2] oct-2-ene with bovine serum albumin by fluorescence spectroscopy," Journal of Luminescence, vol. 128, no. 9, pp. 1454-1458, 2008.

[14] J. Liu, J. Tian, Y. Li, X. Yao, Z. Hu, and X. Chen, "Binding of the bioactive component daphnetin to human serum albumin demonstrated using tryptophan fluorescence quenching," Macromolecular Bioscience, vol. 4, no. 5, pp. 520-525, 2004.

[15] D. Li, M. Zhu, C. Xu, and B. Ji, "Characterization of the baicalein-bovine serum albumin complex without or with $\mathrm{Cu}^{2+}$ or $\mathrm{Fe}^{3+}$ by spectroscopic approaches," European Journal of Medicinal Chemistry, vol. 46, no. 2, pp. 588-599, 2011.

[16] P. Bourassa, I. Hasni, and H. A. Tajmir-Riahi, "Folic acid complexes with human and bovine serum albumins," Food Chemistry, vol. 129, no. 3, pp. 1148-1155, 2011.

[17] S. Ashoka, J. Seetharamappa, P. B. Kandagal, and S. M. T. Shaikh, "Investigation of the interaction between trazodone hydrochloride and bovine serum albumin," Journal of Luminescence, vol. 121, no. 1, pp. 179-186, 2006.

[18] M. Guo, W. J. Lü, M. H. Li, and W. Wang, "Study on the binding interaction between carnitine optical isomer and bovine serum albumin," European Journal of Medicinal Chemistry, vol. 43, no. 10, pp. 2140-2148, 2008.

[19] J. R. Lakowicz, Principles of Fluorescence Spectroscopy, Springer, New York, NY, USA, 3rd edition, 2006.

[20] D. C. Carter, "Crystallographic survey of albumin drug interaction and applications in cancer chemotherapy," in Burgers Drug Design and Development, pp. 437-468, John Wiley \& Sons, New York, NY, USA, 7th edition, 2010.

[21] X. F. Liu, Y. M. Xia, and Y. Fang, "Effect of metal ions on the interaction between bovine serum albumin and berberine chloride extracted from a traditional Chinese Herb coptis chinensis franch," Journal of Inorganic Biochemistry, vol. 99, no. 7, pp. 1449-1457, 2005.

[22] M. Xu, Z. R. Ma, L. Huang, F. J. Chen, and Z. Z. Zeng, "Spectroscopic studies on the interaction between $\operatorname{Pr}(\mathrm{III})$ complex of an ofloxacin derivative and bovine serum albumin or DNA," Spectrochimica Acta Part A, vol. 78, no. 1, pp. 503-511, 2011.

[23] A. Sułkowska, M. Maciaźeka, J. Rŏwnicka, B. Bojko, D. Pentak, and W. W. Sułkowski, "Effect of temperature on the methotrexate-BSA interaction: spectroscopic study," Journal of Molecular Structure, vol. 834, pp. 162-169, 2007.

[24] P. Sandip, S. Chabita, and K. D. Subrata, "Influence of galloyl moiety in C-ring of tea catechins on interaction with bovine serum albumin," in International Conference on Chemical, Biological and Medical Sciences (ICCBMS '12), Kuala Lumpur, Malaysia, August 2012.

[25] X. M. He and D. C. Carter, "Atomic structure and chemistry of human serum albumin," Nature, vol. 358, no. 6383, pp. 209-215, 1992.

[26] U. Kragh-Hansen, "Molecular aspects of ligand binding to serum albumin," Pharmacological Reviews, vol. 33, no. 1, pp. 17-53, 1981.

[27] L. Cyril, J. K. Earl, and W. M. Sperry, Biochemists Handbook, E \& FN Epon Led. Press, London, UK, 1961.

[28] B. Valeur and J. C. Brochon, New Trends in Fluorescence Spectroscopy, Springer Press, Berlin, Germany, 6th edition, 1999.

[29] N. Ibrahim, H. Ibrahim, S. Kim, J. P. Nallet, and F. Nepveu, "Interactions between antimalarial indolone- $\mathrm{N}$-oxide derivatives and human serum albumin," Biomacromolecules, vol. 11, no. 12, pp. 3341-3351, 2010.

[30] P. D. Ross and S. Subramanian, "Thermodynamics of protein association reactions: forces contributing to stability," Biochemistry, vol. 20, no. 11, pp. 3096-3102, 1981.

[31] R. Liu, X. Chu, A. Sun, and L. Kong, "Preparative isolation and purification of alkaloids from the Chinese medicinal herb Evodia rutaecarpa (Juss.) Benth by high-speed counter-current chromatography," Journal of Chromatography A, vol. 1074, no. 1-2, pp. 139-144, 2005. 

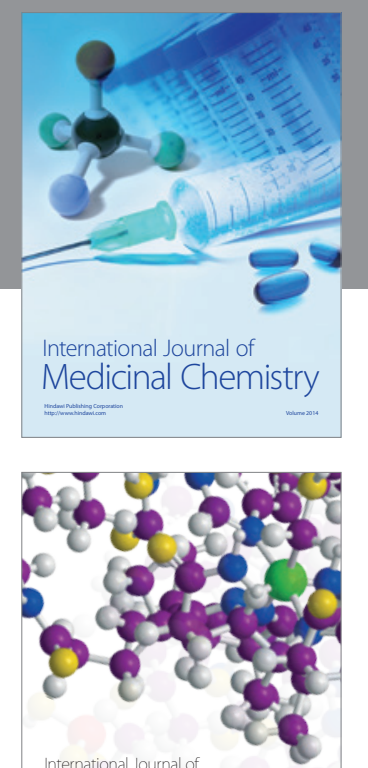

\section{Carbohydrate} Chemistry

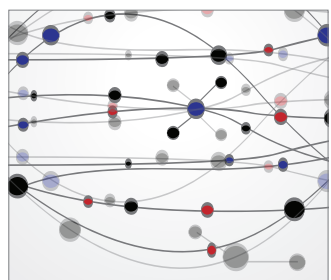

The Scientific World Journal
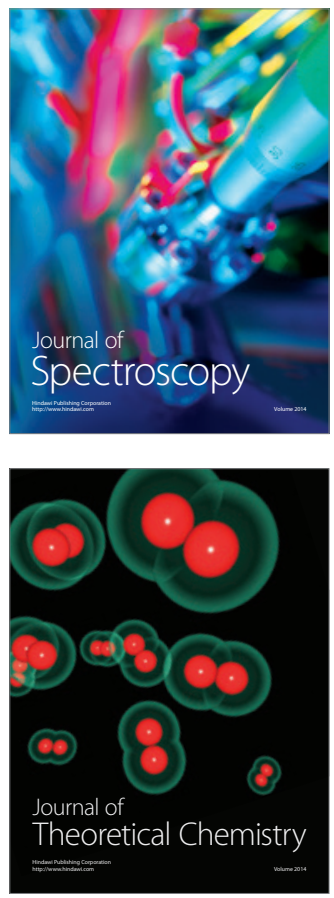
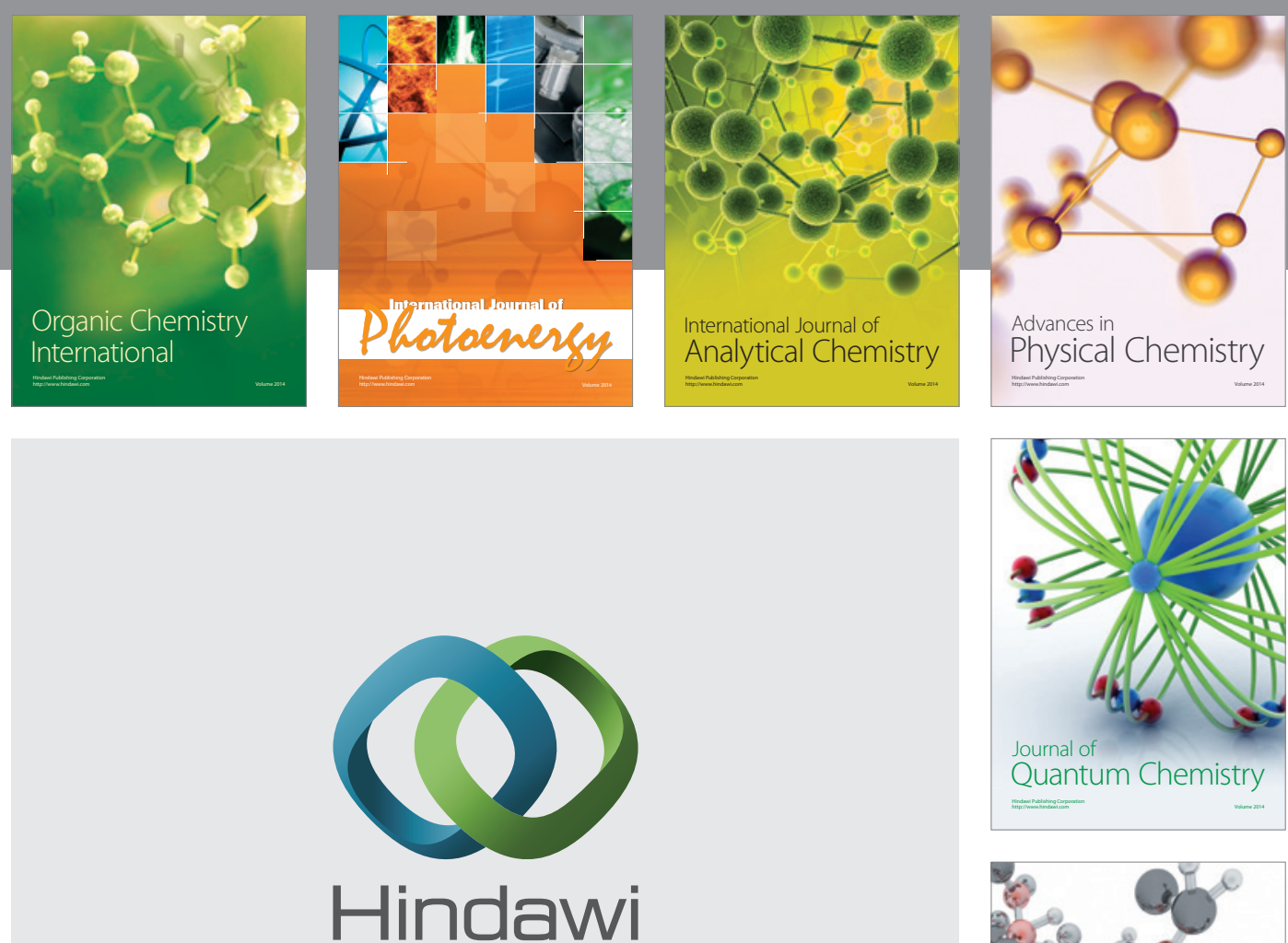

Submit your manuscripts at

http://www.hindawi.com

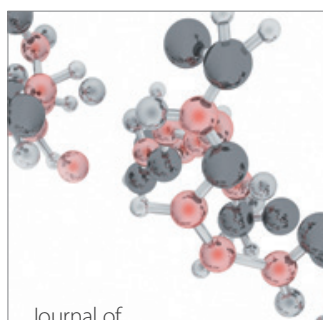

Analytical Methods

in Chemistry

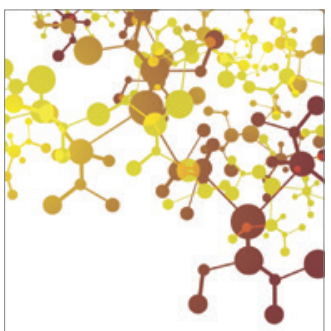

Journal of

Applied Chemistry

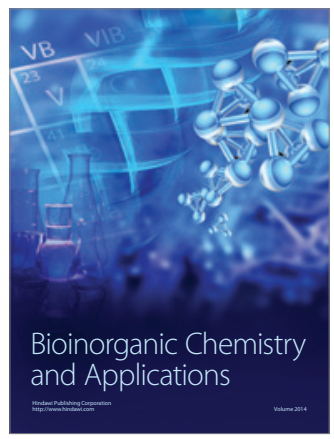

Inorganic Chemistry
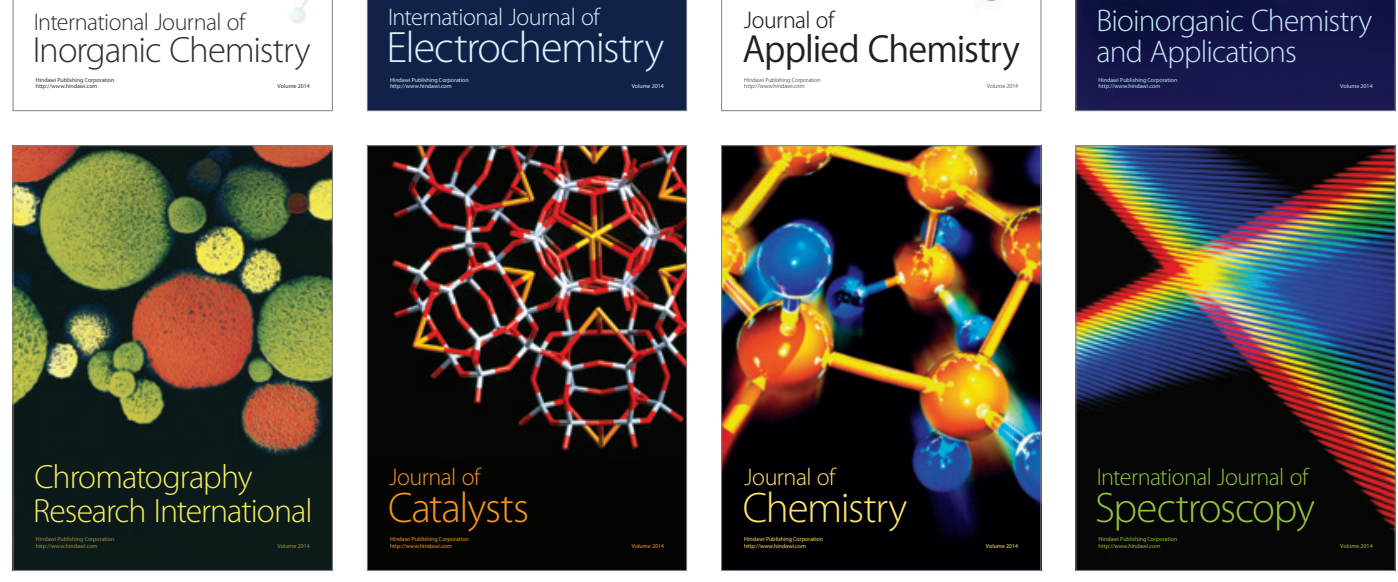